\title{
RACIOCÍNIO COMBINATÓRIO: UMA META-ANÁLISE COM ÊNFASE NAS REPRESENTAÇÕES SEMIÓTICAS
}

\author{
COMBINATORIAL REASONING: A META-ANALYSIS WHIT ENPHASIS ON THE \\ SEMIOTIC REPRESENTATION
}

\author{
Wilian Schmidt \\ Universidade Federal de Santa Maria - wstschmidt@hotmail.com \\ Rita de Cássia Pistóia Mariani \\ Universidade Federal de Santa Maria - rcpmariani@yahoo.com.br
}

\section{Resumo}

Este trabalho objetiva investigar se, e como, são mobilizados os registros de representação semiótica em quatro dissertações stricto sensu produzidas por instituições brasileiras que abordam o desenvolvimento do raciocínio combinatório por meio de atividades didáticas que tiveram a participação de alunos do ensino médio. Para tanto, o estudo embasa-se na meta-análise qualitativa, nos registros de representação semiótica e nas estratégias de resolução de atividades que envolvem raciocínio combinatório. Por meio de dez descritores, a análise dos dados permitiu concluir que o emprego das fórmulas de arranjo, combinação e permutação mobiliza principalmente registros simbólicos e suscita tratamentos nesse mesmo tipo de registro. Nas demais estratégias, como enumeração das configurações solicitadas, recursividade, subdivisão do problema, fixação de variáveis e tradução do problema a outro equivalente, identificou-se uma diversidade maior de registros, a saber: língua natural, figural, tabular e em árvore e, por conseguinte, uma variedade maior nos tipos de conversões entre eles.

Palavras-chave: Raciocínio combinatório; Meta-análise; Registros de representação.

\section{Abstract}

This study aims to investigate whether and how the semiotic representation registers are employed in four strict sense dissertations produced by Brazilian institutions that address the development of combinatory reasoning through educational activities that had the participation of high school students. Therefore the study is based on qualitative metaanalysis in the semiotic representation registers and in solving strategies of combinatorial nature of activities. Through ten descriptors data analysis showed that the use of arrangement, combination and permutation formulas mainly mobilizes symbolic records and raises treatments in the same record type. In other strategies such as enumerating the requested settings, recursion, subdividing the problem, set variables and the translation of the problem in its equivalent there was a greater diversity of records, namely: natural language, figural, tabular and tree, therefore, a greater variety in the types of changes among them.

Keywords: Combinatorial reasoning; Meta-analysis; Register of representation. 


\section{Introdução}

A resolução de atividades que envolvem o raciocínio combinatório é um exemplo de como a Matemática é um campo de estudo que pode se desenvolver por meio de problemas internos, de aplicações ou de necessidades do cotidiano. Guzmán, Batanero e Godino (2001, p. 34) apontam que o raciocínio combinatório é a "base da matemática discreta e, portanto, a raiz de muitos outros ramos da Matemática, como Teoria de Números e Teoria da Probabilidade, e de ciências como Biologia e Economia", pois sua importância está no fato de que "esse modo de pensar está relacionado com quase todas as formas de conhecimentos úteis em que a mente humana pode se ocupar" (BATANERO, GODINO e NAVARRO-PELAYO, 1996, p.17).

Segundo as orientações curriculares dos PCN Ensino Médio, o raciocínio combinatório é uma nova forma de pensar em Matemática que é desenvolvido através da contagem (BRASIL, 2002, p. 126). Além disso, as fórmulas e as expressões que modelam a resolução de problemas denominados por permutação, arranjo e combinação não devem ser entendidas como o único ou principal meio de resolver questões que envolvem combinatória; pois o desenvolvimento do raciocínio combinatório deve estar alicerçado na resolução de problemas, no uso de diferentes representações, na identificação de regularidades e no emprego de modelos matemáticos (BRASIL, 2002, p. 127).

Esse fato está relacionado a um dos objetivos do ensino e aprendizagem dos conceitos relativos aos métodos de contagem e à resolução de suas tarefas: os estudantes devem desenvolver a capacidade de resolver atividades de contagem e de enumeração utilizando diagramas, esquemas de árvores, gráficos, figuras, representações simbólicas, entre outras, ou seja, devem ser capazes de mobilizar diferentes representações matemáticas.

Podemos aproximar as representações matemáticas presentes nas orientações curriculares dos registros de representação semiótica proposto por Duval, ao se considerar que essas diferentes representações matemáticas são meios de acessar os objetos matemáticos e que esses "não são diretamente perceptíveis ou observáveis com a ajuda de instrumentos" (DUVAL, 2003, p.13).

Duval (2009) assume que "um registro de representação é um sistema semiótico que tem as funções cognitivas fundamentais no funcionamento cognitivo consciente". Isso significa que o sujeito não está no "modo automático" ao realizar determinada tarefa, ou seja, ele reflete sobre todos os passos e os procedimentos para executá-la.

Além da finalidade de comunicação, segundo Duval (2003), as representações semióticas são essenciais para as atividades cognitivas do pensamento. Assim, o funcionamento cognitivo do sujeito depende da distinção que ele faz entre o objeto e sua representação e, portanto, está intrinsecamente relacionado com a importância das representações semióticas na matemática e a variedade destas representações.

Nessa perspectiva, procuramos observar como as representações semióticas são empregadas na resolução de atividades que requerem o raciocínio combinatório e, para isso, adotamos a metodologia denominada meta-análise qualitativa porque ela permite 
analisar outros trabalhos pelo viés de um referencial teórico como o dos RRS ${ }^{1}$. Também escolhemos esse método por entendermos que ele valoriza as pesquisas já realizadas sobre o tema que é foco da nossa investigação.

Além disso, a importância dos resultados apresentados nos trabalhos pautados em RRS já foi destacada por Colombo, Flores e Moretti:

[...] o trabalho com registros de representação semiótica com alunos, ou mesmo com professores em processo de formação, possibilita uma melhor compreensão não apenas do objeto matemático em estudo por parte dos estudantes, como também da especificidade da aprendizagem matemática. (COLOMBO; FLORES; MORETTI, 2008, p. 61).

Isso também foi destacado por Curi, Ferreira e Santos (2013, p. 11) ao evidenciarem que as pesquisas sobre RRS se preocupam em "determinar as dificuldades de aprendizagem dos alunos em torno de um tema específico e estabelecer significado de conceitos matemáticos", confirmando, portanto, essa tendência já observada por Colombo, Flores e Moretti (2008, p. 59).

\section{O modelo combinatório implícito $(\mathrm{MCl})$}

As atividades que envolvem o raciocínio combinatório são classificadas por Pessoa e Borba (2010, p. 3) da seguinte forma: produto cartesiano, arranjo, permutação e combinação. Essa classificação é a usualmente adotada e se dá de acordo com características dos objetos que são agrupados: se importa a ordem ou não, se o número de objetos a ser agrupado é igual ou menor do que o número total de objetos do conjunto, se os elementos a serem agrupados provêm de um ou dois conjuntos.

Batanero, Godino e Navarro-Pelayo (1997, p. 239) esclarecem que o desenvolvimento do raciocínio combinatório requer muito mais do que simplesmente resolver problemas de permutação, arranjo e combinação. Isso, porque, segundo os autores, os alunos realizam "atividades de matematização (modelação, representação, formulação, abstração, validação, generalização) quando desenvolvem adequadamente esse modo de pensar".

A identificação da classe dos problemas (arranjo, permutação, combinação, produto cartesiano) pode facilitar a resolução deles, porém, esta não é uma tarefa tão evidente aos alunos. Por isso, Navarro-Pelayo, Batanero e Godino (1996, p. 36) afirmam que as dificuldades referentes à resolução de problemas combinatórios podem estar ligadas a outras variáveis, como, por exemplo, o modelo combinatório implícito (MCl).

Dubois (1984, apud BATANERO; GODINO; NAVARRO-PELAYO, 1996, p. 31) propõe quatro configurações para os problemas de contagem simples que foram classificadas de acordo com os seguintes modelos: seleção (requer a escolha de uma amostra a partir de um conjunto de objetos); distribuição (implica a ordenação de objetos

\footnotetext{
${ }^{1}$ Este artigo está embasado em uma pesquisa de mestrado realizada junto ao Programa de Pós-Graduação em Educação Matemática e Ensino de Física da Universidade Federal de Santa Maria (PPGEMEF/UFSM) que evidenciou uma síntese de investigações stricto sensu produzidas por instituições brasileiras que enfatizaram conceitos do Raciocínio Combinatório desenvolvidos por meio de atividades didáticas com alunos do Ensino Médio.
} 
em caixas, células, urnas, por exemplo); partição (supõe a divisão de objetos de um conjunto em subconjuntos); decomposição (consiste em reescrever um número natural por meio de somas).

Ao adotar essa classificação Batanero, Godino e Navarro-Pelayo (1996, p. 31) explicam que a decomposição é um caso particular da partição reduzindo, assim, 0 número de configurações. Ademais,

[...] estes modelos podem ser considerados como representações ou esquemas concretos inerentes aos enunciados dos problemas combinatórios. A distinção entre estes modelos é importante do ponto de vista matemático, já que o tipo de objetos e representações que intervêm em cada modelo é distinto (amostragem, correspondências, partições de conjuntos, etc.). Isso necessariamente influenciará nos procedimento de resolução e nas dificuldades dos alunos ante as distintas classes de problemas e técnicas de resolução.

Portanto, cada modelo apresenta características específicas, como o tipo de objetos agrupados e a forma como são reunidos. Além disso, por meio do enunciado do problema, é possível associar ações a cada um dos modelos.

De acordo com Navarro-Pelayo, Batanero e Godino (1996, p. 28), os problemas de raciocínio combinatório que possuem palavras-chave como "escolher", "selecionar", "pegar", "extrair", "coletar", podem ser incluídos no modelo de seleção, que tem por característica geral a seleção de $m$ objetos de um conjunto composto de $n$ objetos (distintos em um maior número de casos).

Para ilustrar, pode-se observar o seguinte exemplo: "deseja-se formar uma comissão de três membros e dispõe-se de dez funcionários. Quantas comissões podem ser formadas?". Aqui pode ser observado que formar uma comissão de três membros tem o mesmo significado que "selecionar" três pessoas de uma amostra de dez.

Diferentemente dos problemas de seleção, Navarro-Pelayo, Batanero e Godino (1996, p. 29) esclarecem que as palavras-chave mais comuns no modelo de distribuição são: "colocar", "introduzir", "guardar", "atribuir", entre outras. De forma geral esse modelo engloba os problemas que intencionam a distribuição de $m$ objetos em $n$ caixas (envelopes, urnas, células, etc.).

Para exemplificar esse modelo, destacamos o seguinte problema extraído da mesma obra (1996, p. 37): "dispomos de três cartas iguais. Desejamos colocá-las em quatro envelopes de diferentes cores: amarelo, branco, creme e dourado. Se cada envelope só pode conter, no máximo, uma carta, de quantas formas podemos colocar as três cartas nos quatro envelopes?".

O último modelo é denominado partição e são atribuídas a ele as palavras-chave "dividir", "partir", "decompor", "separar", por exemplo. Esse tipo de problema supõe a divisão de um conjunto de $n$ objetos em $m$ subconjuntos. É possível ilustrar essa situação por meio da seguinte questão: "Maria e Carmen têm quatro figurinhas numeradas de 1 a 4. Decidem reparti-las entre as duas (duas figurinhas para cada uma). De quantas formas podem repartir as figurinhas?". (NAVARRO-PELAYO; BATANERO; GODINO, 1996, p. 38). 
Vale então ressaltar que o modelo combinatório implícito deve ser considerado como uma das variáveis no desenvolvimento do raciocínio combinatório, principalmente por apresentar um diversificado número de atividades de contagem. A partir dessa variabilidade pode-se presumir que existam diversas estratégias que possam ser empregadas para solucionar tais problemas.

\section{Estratégias para a resolução de problemas que envolvem o raciocínio combinatório}

Uma das estratégias que pode ser adotada na resolução de problemas que envolvem o raciocínio combinatório é o diagrama em árvore. Esse procedimento pode ser compreendido como um processo recursivo para a resolução de problemas combinatórios (BATANERO; GODINO; NAVARRO-PELAYO, 1996, p. 65).

Podemos considerar a seguinte situação como exemplo: em uma urna há nove bolas, três vermelhas, três azuis e três verdes. Retirando-se três delas, uma de cada vez, podem-se formar vinte e sete agrupamentos em que alguns podem ser considerados iguais ou distintos a depender do contexto. Tal problema pode ser visualizado e compreendido por meio de um diagrama em árvore (Figura 01).

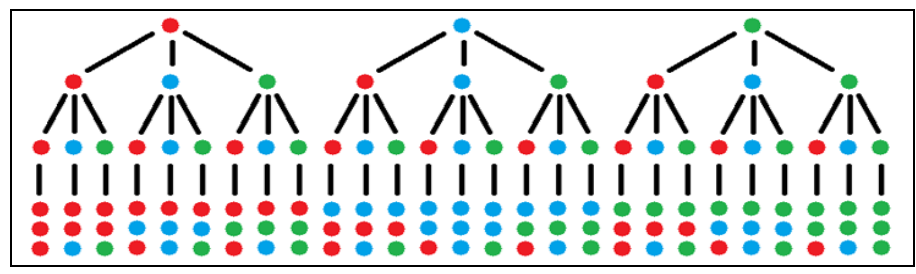

Figura 01 - Diagrama em árvore

Fonte: Autores

Um procedimento é recursivo quando "invoca um versão de si mesmo (em geral com algum câmbio estrutural) como um subprocedimento durante sua execução, ou quando a definição do procedimento contém uma versão de si mesmo como subprocedimento" (BATANERO; GODINO; NAVARRO-PELAYO, 1996, p. 61). Isso revela que um objeto pode ser considerado como recursivo se alguma de suas partes puder ser considerada como cópia de si mesma.

Assim, uma vez observada a recursividade presente nesse procedimento, o que se espera é a generalização do método e que, ao ser utilizado em atividade semelhante, a partir da construção do primeiro ramo do diagrama, seja possível realizar a contagem dos agrupamentos por meio do princípio multiplicativo.

Para resolver um problema combinatório, primeiro é necessário identificar a operação combinatória descrita no enunciado, que geralmente são amostras do tipo ordenada ou não ordenada, com ou sem repetição. Nos problemas de partição ou distribuição, os estudantes devem "fazer uma tradução do problema e formulá-lo em termos de seleção" (GUZMÁN, BATANERO; GODINO, 2003, p. 11).

O aluno ainda pode comparar o problema com outro semelhante no qual conheça a resolução e, após, resolver o original através de analogia. Por exemplo, pode transformar o problema de distribuir três cartas iguais em quatro envelopes diferentes (modelo de 
distribuição) em outro semelhante que consiste em escolher três dos quatro envelopes para inserir as cartas neles (modelo de seleção).

Outra característica principal dos problemas combinatórios é a necessidade de se fixar uma ou mais variáveis para a obtenção de um método coerente de enumeração. Segundo Guzmán, Batanero e Godino (2003, p. 12), "esse não é um método convencional e implica adicionar uma dificuldade a mais aos alunos, visto que eles estão acostumados a usar somente as hipóteses e dados expostos no enunciado do problema".

Esse método consiste na conversão do problema inicial em outro do mesmo tipo, no qual os parâmetros iniciais são reduzidos a outros menores. A partir disso, resolve-se o problema mais simples, para após, resolver o inicial através da recursão.

Por exemplo, se um problema solicita que se combinem quatro pessoas em duplas, pode-se escolher primeiramente duas pessoas e, nesse caso, as outras duas ficam automaticamente determinadas, ou seja, escolhe-se duas das quatro pessoas (primeiro uma e depois a outra). Escolhendo-se uma das quatro (são quatro possibilidades), sobram três, seleciona-se uma dentre as três restantes (são três possibilidades); portanto, pelo princípio multiplicativo, são $4 \times 3=12$ possibilidades.

Guzmán, Batanero e Godino, (2003, p. 13) salientam que os alunos inicialmente tentam resolver os problemas de forma direta e, somente quando não o conseguem, adotam a estratégia de fixar variáveis. Ainda assim, grande parte dos estudantes utiliza essa estratégia corretamente e por esse motivo ela deve ser estimulada na resolução desse tipo de problemas.

Outra estratégia consiste em dividir o problema dado em uma série de problemas menores, resolvê-los independentemente e combinar as soluções parciais para resolver o problema original. Essa forma de resolução de problemas combinatórios é denominada decomposição em subproblemas e supõe:

[...] a decomposição do problema em vários outros, de estrutura combinatória mais simples e parâmetros de menor tamanho, que cobre de maneira exaustiva todos os casos do problema inicial. Combinando adequadamente as soluções parciais, resolve o problema inicial. (GUZMÁN; BATANERO; GODINO, 2003, p. 13).

Como exemplo disso, é possível analisar o problema extraído do mesmo artigo (Ibidem, p. 24): "dispomos de cinco cartas, cada uma delas tem gravada uma letra: A, B, $\mathrm{C}, \mathrm{C}$ e $\mathrm{C}$. De quantas maneiras distintas pode-se colocar na mesa as cinco cartas, uma do lado da outra, formando uma fila?".

Segundo os autores, esse é um problema composto e pode ser resolvido considerando primeiro que as três cartas sejam CCC, e assim as possibilidades seriam CCCAB ou CCCBA. Então a maneira de fixar as três cartas com a letra $C$ são combinações dos cinco lugares tomados 3 a 3, ou seja, 10 possibilidades e como para cada uma delas há duas possibilidades de posicionar as cartas A e B então a solução é dada por 20 possibilidades.

Mesmo havendo essas estratégias para resolução de problemas combinatórios, pode ser que os estudantes não consigam identificar a operação combinatória definida no enunciado. Nesse caso, é possível gerar um modelo combinatório mediante a 
enumeração e contagem, e devem ser empregadas regras de caráter aritmético, tais como, a regra da soma, do produto e do quociente, que, dependendo do tipo de problema, podem ser utilizadas isoladamente ou combinadas entre si.

A regra da soma é adequada para os casos em que o sujeito, não se recordando das fórmulas, tenta gerar um modelo de contagem. Dessa forma, essa regra é utilizada quando "um conjunto de configurações combinatórias se determina como a união de um número de subconjuntos mutuamente exclusivos". (GUZMÁN; BATANERO; GODINO, 2003, p. 15).

Segundo os pesquisadores (2003, p.22), é possível observar a seguinte situação que requer o uso dessa regra: um grupo de quatro amigos têm de realizar dois trabalhos diferentes, um de Matemática e outro de Línguas. Se vão realizar os trabalhos em duplas, de quantas maneiras é possível formar as duplas?

É possível resolver esse problema através da regra da soma: levando em conta que os amigos sejam $A, B, C$ e D, consideram-se os casos em que: $A$ faz o trabalho de Matemática, então há três possibilidades: $A B, A C$ ou $A D ; A$ não faz o trabalho de Matemática, a dupla pode ser: $A B, A C$ ou $A D$. Logo é possível formar seis duplas para a execução das tarefas.

Tal resposta soa coerente, visto que a atividade sugere que os quatro amigos (dispostos em duplas) devem realizar dois trabalhos distintos, ou seja, cada dupla faz um trabalho. Além disso, o método acima não enumera todas as possibilidades de duplas, uma vez que não é necessário, visto que se $A$ forma par com $B$ então não forma com $C$ ou $\mathrm{D}$, além disso, $\mathrm{A}$ fazer Matemática é equivalente a não fazer Línguas, que poderia ser executado apenas por $\mathrm{BC}, \mathrm{BD}$ ou $\mathrm{CD}$.

A regra do produto é outro princípio combinatório de caráter aritmético, no qual são constituídos produtos cartesianos de conjunto de elementos. Seu uso está associado à resolução de problemas compostos e também à geração de um modelo de contagem, em vez do uso de fórmulas.

A regra do produto está ilustrada por meio do problema encontrado em Guzmán, Batanero e Godino, (2003, p. 22): "uma criança tem doze cartas: nove delas são os números 1, 2, 3, 4, 5, 6, 7, 8 e 9. As três restantes são as figuras: valete $(V)$, cavalo (C) e rei $(R)$. De quantas maneiras pode-se alinhar quatro das doze cartas, com a condição de que sempre se tenha três figuras?".

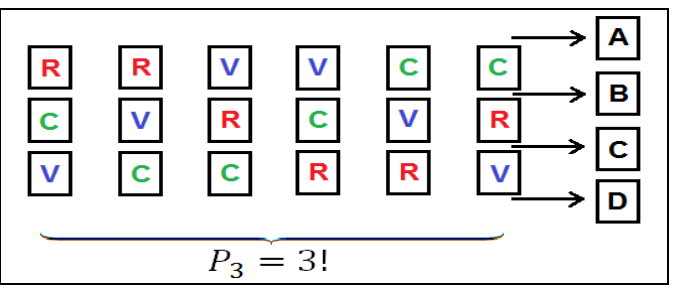

Figura 02 - Esquema de permutação com regra do produto Fonte: Autores

$\mathrm{Na}$ Figura 02, pode-se observar que cada coluna representa as permutações das cartas com figuras (3!), como devem ser alinhadas quatro cartas, a que contém o número 
pode ser posta na posição $A, B C$ ou $D$ e isso deve ocorrer para cada uma das seis colunas (3!*4). Por fim, as cartas numéricas podem ser escolhidas de nove modos diferentes $(3 ! * 4 * 9)$. Logo, a solução do problema é dada por $3 ! * 4 \times 9=216$ maneiras diferentes.

Para relacionar entre si combinações e arranjos ou permutações simples e permutações com repetições, é necessário o uso da regra do quociente. Segundo Guzmán, Batanero e Godino (2003, p. 18), "usar a regra do quociente implica estabelecer uma relação de equivalência dentro de um conjunto de configurações combinatórias".

Além de estabelecer a relação de equivalência dentro de um conjunto de configurações combinatórias, é necessário identificar o número de elementos em cada classe de equivalência. Esse fato pode se percebido no exemplo: Em uma caixa, há duas fichas azuis, uma branca e uma vermelha. As quatro fichas são retiradas da caixa ao acaso e sem reposição, anotando-se a cor a cada vez. De quantas maneiras diferentes é possível fazer a seleção das fichas? (GUZMÁN; BATANERO; GODINO, 2003, p. 21).

Para resolver esse problema, é preciso observar que a ficha azul aparece duas vezes em cada agrupamento. Apesar disso, cada vez que são permutadas apenas as fichas azuis, não há a formação de um agrupamento distinto, como pode ser visto nas linhas 1 e 2 da figura 03. Entretanto, será formado um agrupamento diferente quando houver troca de fichas de cores distintas de posição (linha 3). Assim, o problema pode ser resolvido utilizando a regra do quociente como segue: $P_{4} / 2 !=12$ maneiras distintas.

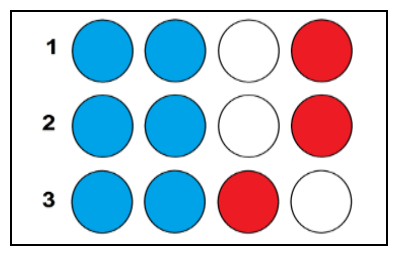

Figura 03 - Esquema de permutação com repetição

Fonte: Autores.

De modo geral, segundo Guzmán, Batanero e Godino, (2003, p. 20), é possível observar que se manifestam inoperantes as regras da soma e quociente; ao contrário, a regra do produto se mostra uma ferramenta "popular" entre os estudantes na resolução de problemas combinatórios. Acreditamos que isso ocorra porque os problemas do tipo produto cartesiano, normalmente, são os primeiros a serem explorados no desenvolvimento do raciocínio combinatório.

O emprego de estratégias para resolver atividades de contagem está atrelado a fatores como o modelo ao qual pertence o problema, a operação combinatória associada a ele e ao tamanho dos parâmetros $m$ e $n$. Cremos que adoção desses métodos favorece o desenvolvimento do raciocínio combinatório e reduz incompreensões causadas pelo uso exclusivo de fórmulas.

Assim, nas análises realizadas neste trabalho, foram averiguadas as estratégias adotadas na resolução de problemas combinatórios. Buscamos entender como essas estratégias são empregadas de acordo com a variabilidade das tarefas (seleção, partição 
e distribuição), bem como das operações combinatórias básicas (permutação, arranjo e combinação).

\section{Representações semióticas no raciocínio combinatório}

Para Duval (2003, p.11), um dos principais objetivos da aprendizagem da matemática é contribuir para o desenvolvimento geral das capacidades de raciocínio, de análise e de visualização dos alunos. Pode parecer simples alcançar esse objetivo, mas deve-se considerar que os objetos matemáticos se tratam essencialmente de conceitos, propriedades, estruturas, relações que podem expressar diferentes situações, entre outros. Por isso, os objetos de outras áreas do conhecimento são diferentes dos da Matemática.

Para acessar os entes matemáticos, é necessário que eles sejam evidenciados por meio de gráficos, figuras, tabelas, expressões, fórmulas, por exemplo. Essas formas de expressar os objetos são denominadas representações matemáticas.

Há uma grande variedade de representações que são utilizadas em matemática, dentre as quais se podem citar: os diferentes sistemas de numeração, as figuras geométricas, as escritas algébricas e formais, as representações gráficas e a língua natural. Duval (2003) aponta restrições de se utilizar um único registro semiótico para representar um mesmo objeto matemático uma vez que "a compreensão em Matemática implica a capacidade de mudar de registro”. (DUVAL, 2003, p. 21).

Dessa forma, ele defende que não se deve jamais confundir um objeto e sua representação, uma vez que, operando em mais de um sistema de representação, é implícito e primordial o entendimento de que nenhum dos registros de representação "é" o objeto matemático, mas que apenas o "representa".

Para designar os diferentes tipos de representações semióticas empregados em matemática, Duval (2003, p. 14) adotou o termo registro e os classificou em quatro tipos: os registros multifuncionais discursivos e não discursivos e os registros monofuncionais também divididos em representações discursivas e não discursivas (veja o Quadro 01 a seguir).

Portanto, quando se quer analisar as atividades matemáticas sob a perspectiva da aprendizagem, é importante considerar todos os tipos de representações. É essencial analisar a mobilização dos diversos tipos de registros, e não de um só. Essa classificação proposta por Duval atende às transformações dos registros de representação de muitos objetos matemáticos.

A noção de RRS permite salientar a importância da mudança de registros e a necessidade de uma coordenação entre eles. Existem dois tipos de transformações de representações semióticas: os tratamentos e as conversões. (DUVAL, 2003, p. 15).

Segundo Duval (2009, p.39), o tratamento é a transformação de uma representação em outra representação de um mesmo registro, por isso é considerado como uma "modificação estritamente interna a um determinado registro". Os tratamentos são ligados mais à forma do que ao conteúdo, no sentido de que um mesmo objeto 
matemático pode ter mais de uma representação diferente. Nesse caso, apresenta tratamentos também diferenciados com graus de dificuldade diversos.

Quadro 01 - Classificação dos Registros de Representação Semiótica a partir do objeto matemático desta pesquisa.

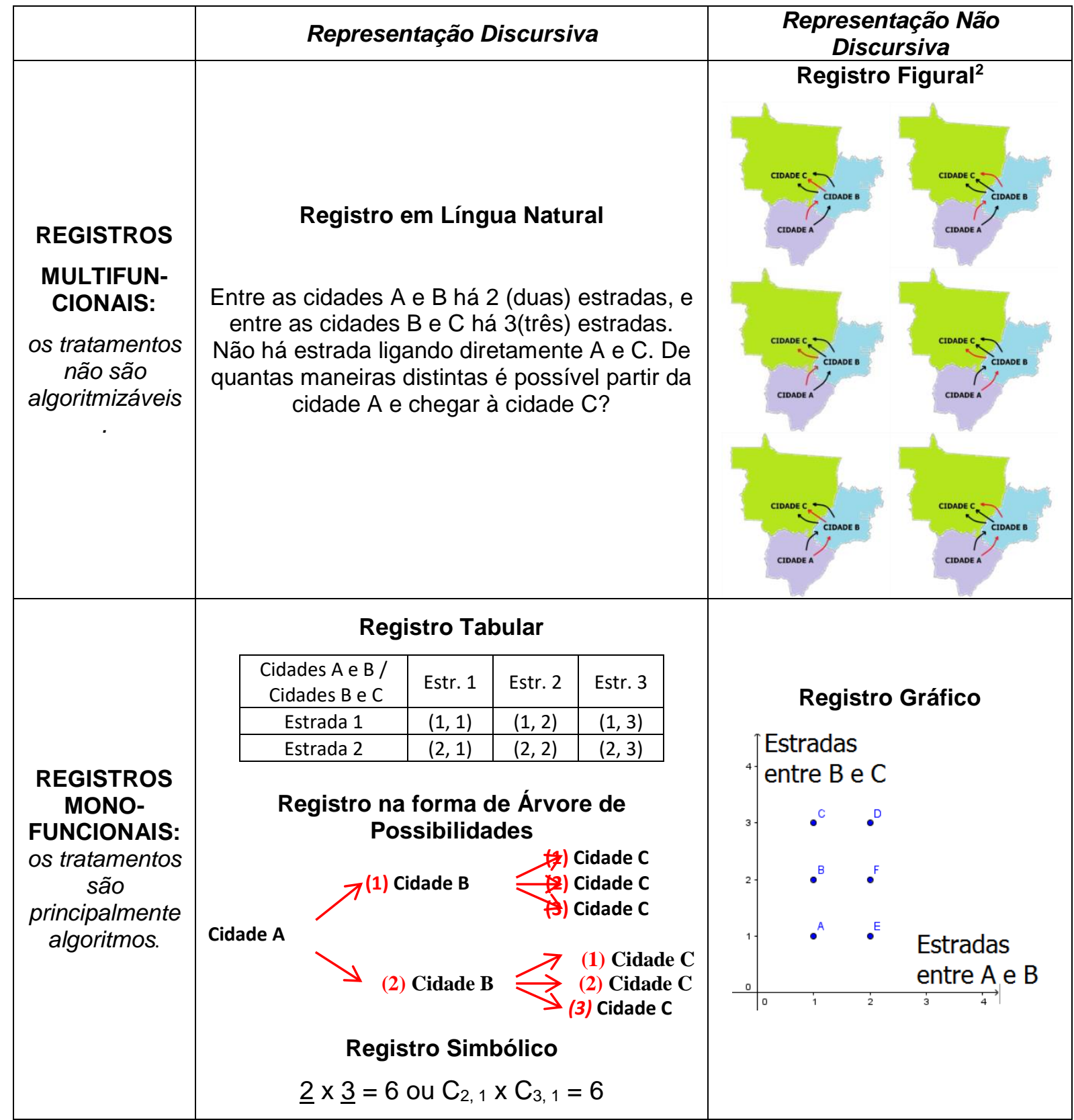

Fonte: Adaptado pelos autores (DUVAL, 2003, p. 14).

O registro simbólico apresenta uma transformação interna quando o problema é resolvido através da combinação: $C_{2,1} \times C_{3,1}=6$. O tratamento, nesse caso, efetua-se por meio da operação de multiplicação.

Enquanto isso, as conversões são transformações que ocorrem entre registros diferentes. A representação de um objeto em um registro específico é convertida em uma

\footnotetext{
${ }^{2}$ Nesse caso, o registro figural não possui propriedades geométricas, e, portanto, não pode ser caracterizado por meio dos pressupostos que Duval (2012a e 2012b) utiliza para analisar a aprendizagem em Geometria.
} 
representação de outro registro, que conserva a referência, mas não conserva a explicitação das mesmas propriedades desse objeto. Assim, o sentido da representação do objeto em um registro de partida não será o mesmo do registro de chegada.

\section{Aspectos metodológicos}

Para atender ao objetivo proposto, tomamos a meta-análise qualitativa como metodologia por se tratar de uma "revisão sistemática de outras pesquisas, visando realizar uma avaliação crítica delas e/ou produzir novos resultados ou sínteses a partir do confronto desses estudos, transcendendo aqueles anteriormente obtidos" (FIORENTINI e LORENZATO, 2006, p. 103). Os propósitos desse método são: reunir resultados ou sínteses interpretativas de diferentes pesquisas com o intuito de alcançar um nível teórico mais elevado em relação ao que seria obtido com apenas um estudo; preencher os conceitos abstratos de um estudo com significados explicitados em sínteses de resultados de outros; e, atentar para o desenvolvimento teórico dos resultados analisados de um tema que é descritivo e compreensivo, logo, mais completo do que qualquer estudo sozinho (BICUDO, 2014, p. 11).

Como o objetivo deste trabalho era "investigar se e como são mobilizados os RRS nas investigações stricto sensu produzidas por instituições brasileiras que abordam o desenvolvimento do raciocínio combinatório por meio de atividades didáticas que tiveram a participação de alunos do ensino médio", dividimos a pesquisa em etapas, nas quais os procedimentos adotados são: mapeamento de dissertações e teses que abordam raciocínio combinatório; definição dos critérios de seleção dos trabalhos que constituirão o corpus documental da meta-análise; definição das categorias de análise, as quais denominamos de "descritores"; por fim, pautados nos descritores, apresentação da análise dos dados obtidos a partir dos trabalhos selecionados.

O primeiro passo, ou seja, o mapeamento de teses e dissertações brasileiras que abordam o raciocínio combinatório ocorreu por meio das palavras-chave "análise combinatória", "combinatória" e "permutações" nos sites dos programas de pós-graduação de universidades brasileiras (grande área Multidisciplinar, área de Ensino de Ciências e Matemática) e no Banco de Teses e Dissertações da Coordenação de Aperfeiçoamento de Pessoal de Nível Superior (CAPES).

Foi possível catalogar 43 pesquisas assim distribuídas: 33 dissertações de mestrado profissionalizante, 06 dissertações de mestrado acadêmico e 04 teses de doutorado. Esse mapeamento inclui somente as pesquisas disponibilizadas online nos sites dos programas de pós-graduação.

Após a primeira leitura e análise das pesquisas, selecionamos os trabalhos que abordavam o raciocínio combinatório no ensino médio, tanto do ensino em idade regular quanto na modalidade de educação de jovens e adultos, porque acreditamos que 0 desenvolvimento do raciocínio combinatório promove habilidades e competências relacionadas à resolução de problemas que são mais acuradas em estudantes nesse nível de ensino. Além disso, priorizamos as pesquisas que exploraram em seu referencial teórico a resolução de problemas $(\mathrm{RP})$, pois acreditamos que, ao resolver uma situação 
problema, o estudante necessita se amparar em uma diversidade maior de registros de representação.

Nesse contexto, selecionamos quatro investigações (quadro 02) que continham atividades envolvendo raciocínio combinatório. Vale ressaltar que os quatro trabalhos elencados atendem a outro critério, o fato de o autor explicitar e analisar as atividades propostas no âmbito do raciocínio combinatório expondo no seu corpus documental soluções das atividades de alguns alunos.

Quadro 02 - Pesquisas selecionadas

\begin{tabular}{|c|c|c|c|}
\hline Título & Instituição/Ano & Autor(a) & Orientador(a) \\
\hline $\begin{array}{l}\text { Análise combinatória no ensino médio } \\
\text { apoiada na metodologia de ensino- } \\
\text { aprendizagem-avaliação de Matemática } \\
\text { através da resolução de problemas }\end{array}$ & $\begin{array}{l}\text { UNESP/SP } \\
\text { Rio Claro } \\
2010\end{array}$ & $\begin{array}{l}\text { Analucia } \\
\text { Castro } \\
\text { Pimenta de } \\
\text { Souza }\end{array}$ & $\begin{array}{l}\text { Lourdes de La } \\
\text { Rosa Onuchic }\end{array}$ \\
\hline $\begin{array}{l}\text { O Ensino de Análise Combinatória a partir } \\
\text { de Situações-Problema }\end{array}$ & $\begin{array}{l}\text { UEPA/PA } \\
2008\end{array}$ & $\begin{array}{l}\text { Carlos } \\
\text { Alberto de } \\
\text { Miranda } \\
\text { Pinheiro }\end{array}$ & $\begin{array}{l}\text { Pedro Franco } \\
\text { de Sá }\end{array}$ \\
\hline $\begin{array}{l}\text { Análise combinatória na educação de } \\
\text { jovens e adultos: uma proposta de ensino a } \\
\text { partir da resolução de problemas }\end{array}$ & $\begin{array}{l}\text { UFRGS/RS } \\
2012\end{array}$ & $\begin{array}{l}\text { Jussara } \\
\text { Aparecida da } \\
\text { Fonseca }\end{array}$ & $\begin{array}{l}\text { Elisabete } \\
\text { Zardo Búrigo }\end{array}$ \\
\hline $\begin{array}{l}\text { Uma investigação no ensino médio sobre o } \\
\text { raciocínio combinatório e a divergência de } \\
\text { resultados na resolução de problemas de } \\
\text { contagem }\end{array}$ & $\begin{array}{l}\text { UNIVATES/RS } \\
2014\end{array}$ & $\begin{array}{l}\text { Roberto } \\
\text { Stenio Areias } \\
\text { Carneiro de } \\
\text { Albuquerque }\end{array}$ & $\begin{array}{l}\text { Claus } \\
\text { Haetinger }\end{array}$ \\
\hline
\end{tabular}

Fonte: Autores.

Ao realizar a primeira leitura das dissertações selecionadas, observamos que Souza (2010) e Pinheiro (2008) apresentam possíveis soluções que esperam obter dos participantes na resolução das atividades propostas em seus trabalhos. Além disso, Fonseca (2012) explora com os estudantes um jogo que aborda o raciocínio combinatório e Albuquerque (2014) apresenta em seus anexos uma série de atividades que foram desenvolvidas com os estudantes, mas não fizeram parte das análises.

Desse modo, optamos por analisar somente as atividades resolvidas pelos estudantes e que requeriam o raciocínio combinatório em sua solução. Assim, não analisamos as resoluções apresentadas pelos autores ou quaisquer outras atividades que não tenham caraterísticas de problemas combinatórios.

As atividades provenientes das dissertações foram analisadas em três etapas, sendo que a primeira delas consiste em classificar os problemas de acordo com o modelo combinatório implícito: problemas de seleção (escolha de uma amostra a partir de um conjunto de objetos), distribuição (ordenação de objetos em caixas, células, urnas) ou partição (divisão de objetos de um conjunto em subconjuntos).

A segunda etapa se caracterizou por identificar a presença das categorias de análise (descritores) em cada uma das resoluções das atividades. Por fim, realizamos a classificação dos tipos de registros presentes em cada uma delas, especificamente na partida (enunciado), no intermediário (resolução) e na chegada (resposta). 
Adotamos nessa classificação as seguintes notações: registro em língua natural $(R L N)$, registro tabular (RTb), registro em árvore (RAv), registro figural (RFg), registro simbólico $(R S b)$ e registro gráfico $(R G f)$. Utilizamos uma seta $(\rightarrow$ ) para identificar o sentido dos registros mobilizados, por exemplo, $\mathrm{RSb} \rightarrow \mathrm{RTb}$ representa a identificação tanto do registro simbólico quanto do tabular em uma mesma resolução, sendo o registro simbólico identificado no enunciado e o registro tabular na resolução, sem necessariamente implicar em uma conversão entre os dois registros. Utilizamos, ainda, dois pontos (:) quando houve uma confirmação da resolução, por exemplo, se a resolução se dá por meio do registro simbólico e é confirmada pelo tabular, então expressamos esse fato por RSb:RTb.

Com o objetivo de aprofundamento da análise das atividades, elaboramos descritores (Quadro 03) selecionando elementos que consideramos importantes no desenvolvimento do raciocínio combinatório e, para isso, baseamo-nos no aporte teórico sobre estratégias de resolução de problemas que envolvem o raciocínio combinatório.

\section{Quadro 03 - Descritores}

\begin{tabular}{|l|l|}
\hline 01 & Utilizar fórmula de permutação, arranjo ou combinação. \\
\hline 02 & Ocupar a regra do produto. \\
\hline 03 & Formar as configurações pedidas no enunciado do problema. \\
\hline 04 & $\begin{array}{l}\text { Recorrer à recursividade como ponto de apoio para desenvolver estratégias de } \\
\text { solução. }\end{array}$ \\
\hline 05 & Decompor o problema inicial em subproblemas. \\
\hline 06 & Usar a regra da soma. \\
\hline 07 & Fixar variáveis. \\
\hline 08 & Traduzir o problema a outro equivalente. \\
\hline 09 & Empregar a regra do quociente. \\
\hline 10 & Enumerar não sistematicamente os agrupamentos. \\
\hline
\end{tabular}

Fonte: Autores.

Tais descritores nos auxiliaram a padronizar a análise das atividades, pois, em cada estratégia de resolução, identificamos a presença dos descritores e, em cada um deles, os registros de representação mobilizados.

Assim, esclarecemos que esta lista de descritores não tem caráter prescritivo, tratase apenas de uma relação de elementos teóricos considerados importantes que usamos como base para as análises. Portanto, seria necessário um estudo mais aprofundado sobre quais deles seriam convenientes para ser explorado pelo professor em sala de aula.

\section{Discussão dos resultados}

Após analisar as resoluções das atividades presentes nas dissertações selecionadas, observamos que em todas as atividades foi identificado pelo menos um descritor, sendo que em muitas foram identificados três ou mais descritores. Os descritores 02,03 e 05 foram os observados com maior frequência na resolução das tarefas, $80 \%, 47,5 \%$ e $47,5 \%$ respectivamente.

Além disso, o descritor 01 não foi identificado na dissertação de Souza (2010), ou seja, não foram utilizadas expressões algébricas e/ou fórmulas pelos estudantes na 
resolução dos problemas propostos. Uma hipótese para que isso tenha ocorrido embasase no fato de que os estudantes resolveram as atividades antes das plenárias serem realizadas. A intenção era a de formalizar os conhecimentos de permutação, arranjo e combinação, e, quando foi possível a discussão das resoluções presentadas pelos grupos, eles eram indagados sobre a quantidade de elementos nos agrupamentos e sobre a importância da ordem deles. Tais indagações visavam a reflexões que tinham a intenção de estimular a formalização de conceitos combinatórios (arranjo, permutação e combinação).

Também, talvez pelo mesmo motivo, o descritor 07 não foi identificado na realização das atividades apresentadas pelos estudantes. Entretanto, a autora previu em seu projeto inicial que os participantes poderiam utilizar a estratégia de fixar variáveis como método de resolução em uma das atividades propostas.

As análises das atividades da pesquisa de Souza (2010) apontam que os participantes do estudo utilizam com maior frequência a regra do produto e a formação de configurações solicitadas no enunciado para resolver problemas combinatórios. Frequentemente foram utilizados os registros do tipo tabular e figural na resolução das sete atividades propostas e, com menos regularidade, os do tipo árvore e simbólico. Entretanto, as operações do tipo tratamento tiveram um maior destaque no registro simbólico. Como as atividades apresentam registro de partida em língua natural, foi possível observar nesses descritores a mobilização dos seguintes registros: $R L N \rightarrow R T b$, $\mathrm{RLN} \rightarrow \mathrm{RSb}, \mathrm{RLN} \rightarrow \mathrm{RAv}, \mathrm{RLN} \rightarrow \mathrm{RFg}$. Além disso, a maior parte dos registros de chegada (respostas) são em língua natural.

Os descritores 04 e 05 também estão presentes nas resoluções das atividades propostas por Souza (2010). Alguns participantes da pesquisa utilizaram a recursividade para resolver os problemas. Nesse método, pudemos identificar $R L N \rightarrow R A v$ e $R L N \rightarrow R f g$, sendo que os tratamentos foram executados no registro simbólico. Enquanto isso, alguns grupos utilizaram a estratégia da decomposição em subproblemas e, para tal, uma diversidade maior de registros do tipo RTb, RAv, RFg e RSb.

Muito menos frequentes foram identificados os descritores 06, 08, 09 e 10, ou seja, o uso da regra da soma, a tradução do problema a outro equivalente, o uso da regra do produto e enumeração não sistemática não chegam a configurar um padrão de resolução de problemas nessa dissertação. Desse modo uma menor diversidade de registros foi identificada nesses descritores, podendo-se destacar RTb, RAv, RFg e RSb.

Por fim, cabe ressaltar que em apenas uma atividade foi identificado um único descritor. As outras seis atividades apresentaram mais de um descritor nas resoluções propostas pelos estudantes; foram identificados isoladamente ou em associação. Um exemplo disso é o emprego da recursividade, em que primeiro foram formadas algumas configurações do enunciado e, a partir dessa estratégia atingir, por meio da regra do produto ou soma, a resposta desejada.

A dissertação de Pinheiro (2008) propunha treze atividades das quais nove são do tipo seleção e quatro do tipo distribuição. Não houve a presença dos descritores 07 e 08 na resolução apresentada pelos participantes, respectivamente, fixar variáveis e tradução do problema a outro equivalente. 
A primeira observação a ser feita é que somente nessa pesquisa foi identificada a presença de expressões ou fórmulas como meio de obtenção do resultado exigido no enunciado. Não nos surpreendemos ao observar que em todas as resoluções houve mudanças nas representações no sentido RLN $\rightarrow R S b$ e, também, tratamentos no registro simbólico. As respostas foram dadas por meio de registro em língua natural e registro simbólico. Além disso, há nesste, a presença conjunta do descritor 09.

Com exceção de uma atividade, em todas as demais (doze), foi identificada a regra do produto nas soluções apresentadas pelos participantes, ou seja, o descritor 02 esteve presente em quase todas as atividades. Foi possível observar a mobilização dos seguintes registros de partida e chegada: $R L N \rightarrow R F g, R L N \rightarrow R T b, R L N \rightarrow R A v$ e $\mathrm{RLN} \rightarrow \mathrm{RSb}$, mas também foram identificadas alterações nos registros intermediários no sentido RFg $\rightarrow R S b$. Em todas as atividades, há a presença do registro simbólico como intermediário, no qual ele configura o cerne da resolução ou é mobilizado para auxiliar a obtenção da solução por meio de outro tipo de registro.

O descritor 03 foi utilizado de maneiras diversas como estratégia de solução dos problemas. Alguns participantes formam as configurações solicitadas no enunciado para apoiar uma resolução por meio da regra do produto, outros como método principal de solução, e, por fim, há aqueles que utilizaram esse método para verificação da resolução por meio de outra estratégia. Mesmo assim, foram identificadas as mobilizações $R L N \rightarrow R T b$ e $R L N \rightarrow R S b$, o que justifica uma presença maior dos registros do tipo RTb e $\mathrm{RSb}$ nos registros intermediários. Ainda observamos que todos os registros de chegada são em língua natural.

A recursividade foi utilizada como estratégia de resolução de problemas em duas atividades distintas, sendo que, ou foi utilizada como método principal ou como confirmação da resolução produzida por meio da regra do produto. Portanto, em uma das estratégias, foi considerada a mudança no sentido $R L N \rightarrow R A v$, enquanto na outra não foi considerada por se tratar apenas de uma confirmação. Assim, os registros intermediários identificados são do tipo árvore e simbólico.

Os descritores 05 e 06 são utilizados, de maneira geral, como apoio para outros métodos e como finalização da resolução, isto é, a decomposição em subproblemas facilita a organização dos dados e a regra da soma é utilizada para a conclusão do resultado. Entretanto, alguns participantes também os utilizaram de forma isolada, gerando, dessa forma, respostas incompletas ou equivocadas. Foi possível identificar modificações de registros nos sentidos $R L N \rightarrow R A v$ e $R L N \rightarrow R F g$. Hás assim, uma predominância dos registros em árvore, figural e simbólico nesses descritores.

Assim, como na pesquisa de Souza (2010), o descritor 10 é pouco distinguível, fazendo-se presente por meio de registros tabulares, figurais ou em língua natural. Também é observável a associação de vários descritores em uma mesma atividade. Como é o caso do uso de expressões algébricas que envolvem, em muitos casos, simultaneamente a regra do produto e quociente.

Um dos objetivos do trabalho de Fonseca (2012) era propor aos participantes a realização de atividades que não dependessem do conhecimento prévio de expressões 
algébricas para a sua resolução. Desse modo, não é de se estranhar que o descritor 01 não tenha sido identificado nas resoluções das atividades.

Com exceção da atividade 23, em todas as demais, foi possível identificar o descritor 02, ou seja, em quase todas as atividades algum dos participantes resolveu 0 problema por meio da regra do produto. Os registros de partida e os registros intermediários são do tipo $R L N \rightarrow R S b, R L N \rightarrow R A v$ e $R L N \rightarrow R T b$. Por consequência, também é possível identificar as mudanças nos sentidos $R A v \rightarrow R S b$ e $R T b \rightarrow R S b$. Portanto, os registros que se destacam são os do tipo RSb, RAv e RTb, sendo que os tratamentos são essencialmente no registro simbólico. As atividades nas quais foi identificada a presença do descritor 02 são em sua grande maioria problemas do tipo seleção.

Os descritores 03, 04, 05 e 06 são mobilizados na mesma perspectiva em relação à dissertação de Pinheiro (2008). Com exceção do descritor 03, em que foi possível ser observado nos problemas do tipo seleção, distribuição e partição, os demais (descritores 04, 05 e 06) foram identificados essencialmente em atividades do tipo seleção. Em relação aos registros mobilizados, o descritor 06 , por se tratar da regra da soma, mobiliza basicamente o registro simbólico com tratamentos neles. Já os demais descritores apresentam uma diversidade maior de registros, sendo eles dos tipos RLN, RTb, RAv, RFg e RSb.

Enquanto isso, o descritor 07 foi identificado apenas na dissertação de Fonseca (2012) e, apesar de o seu uso não ter sido totalmente adequado, pois alguns grupos não utilizaram recorrência para concluir os resultados, foi possível observar mesmo em apenas três atividades, a presença dos registros de representação. Algumas modificações presentes em certas resoluções se dão no sentido $R L N \rightarrow R F g$ e $R L N \rightarrow R A v$. Assim, os registros empregados são do tipo $R L N$, RFg, $R A v$ e $R S b$, sendo que os tratamentos ocorreram no simbólico.

O descritor 10 esteve pouco presente nas resoluções apresentadas pelos participantes da pesquisa de Fonseca (2012). Ele foi identificado em apenas duas atividades, sendo que, em uma delas, a enumeração não sistemática serviu como estratégia principal de resolução no registro figural e foi confirmado por meio de enumeração sistemática por meio de registro tabular. Enquanto isso, na outra, o procedimento não foi utilizado de modo adequado, sendo apresentado por meio do registro em língua natural.

As atividades propostas na pesquisa de Albuquerque (2014) se dividem em três problemas de seleção e dois problemas de partição. As questões dessa dissertação estão divididas em pré-teste e pós-teste, sendo em ambas as categorias os mesmos problemas. Por esse motivo, incluímos os subíndices "a" e "b" na análise das atividades. Nesse trabalho, foi possível identificar cinco dos dez descritores, a saber: 02, 03, 05, 06 e 10. Isso quer dizer que foram identificadas as estratégias de regra do produto, formação das configurações solicitadas, decomposição do problema inicial em subproblemas, regra da soma e enumeração não sistemática. Tais estratégias foram utilizadas de modo semelhante em relação às demais dissertações. Entretanto, tais resoluções não apresentam uma diversidade no tipo de registros empregados, nem nos intermediários, 
nem nos de chegada. Os mais usuais foram RSb e RFg para os intermediários e RLN nos de chegada. Dessa forma, as alternâncias entre o registro de partida e o intermediário são dos tipos $\mathrm{RLN} \rightarrow \mathrm{RSb}$ ou $\mathrm{RLN} \rightarrow \mathrm{RFg}$. Concluímos que não houve uma modificação significativa dos registros empregados na resolução das atividades do pré-teste e nas apresentadas na resolução dos problemas do pós-teste.

Em relação ao $\mathrm{MCl}$, é possível concluir que $75 \%$ das atividades são problemas do tipo seleção, sendo que 10\% dessas foram identificadas na dissertação de Souza (2010), $30 \%$ na pesquisa de Pinheiro (2008), 43,3\% no estudo de Fonseca (2012) e 16,7\% no trabalho de Albuquerque (2014). Sobre essas questões do tipo seleção, temos que: duas podem ser resolvidas por meio de arranjo com repetição; quatro com o auxílio de combinação; onze como se fosse arranjo e treze são do tipo composto (não podem ser resolvidas por meio de uma única fórmula).

Identificamos seis problemas do tipo distribuição, sendo que dois deles são compostos, um pode ser interpretado como uma aplicação injetiva (arranjo) e três podem ser concebidos como aplicação bijetora (permutação). Contabilizamos apenas quatro problemas como sendo do tipo partição.

Em relação aos descritores, o 02 (que se refere ao uso da regra do produto) foi verificado em maior frequência. Ainda vale ressaltar que, em 55\% das atividades, há a presença de três ou mais descritores, o que significa haver uma grande diversidade no emprego de estratégias de resolução de problemas combinatórios (regra do produto, enumeração das configurações, recursividade, decomposição em subproblemas e regra da soma).

Com a análise das atividades por meio dos descritores, foi possível concluir que a resolução dos problemas por meio de fórmulas de permutação, arranjo e combinação, ao contrário do que imaginávamos, não configurou uma escolha óbvia pelos participantes das pesquisas, ou seja, essa estratégia não foi amplamente empregada. Quando utilizada, esteve acompanhada da regra do quociente e/ou regra do produto. Sendo assim, o registro simbólico é privilegiado no descritor 01.

Pudemos confirmar a popularidade da regra do produto na resolução dos problemas combinatórios (GUZMÁN; BATANERO; GODINO, 2003), uma vez que o descritor 02 se fez presente nas resoluções de 32 (trinta e duas) das 40 (quarenta) atividades analisadas. Novamente, por se tratar de uma estratégia que envolve "produtos" numéricos, o registro de representação característico desse descritor é o simbólico. Em contrapartida, apesar de o descritor 09 (regra do quociente) também ser caracterizado pelo registro simbólico, foi identificado nas resoluções de apenas seis atividades.

Enquanto isso, enumeração das configurações solicitadas (descritor 03), recursividade (descritor 04), decomposição do problema inicial em subproblemas (descritor 05) e regra da soma (descritor 06) apareceram de forma isolada ou em conjunto na resolução das atividades. Esses descritores apresentaram uma diversidade grande em relação aos registros de representação semiótica.

Dentre as estratégias de resolução de problemas combinatórios, as menos empregadas foram a de fixar variáveis (descritor 07) e a tradução do problema a outro 
equivalente (descritor 08), em que vale destacar a presença dos registros em árvore, simbólico e figural no descritor 07 e o registro figural no descritor 08 . Outra estratégia não muito empregada foi a de enumeração não sistemática (descritor 10), que na maioria das atividades configura um uso não bem sucedido, o qual atribuímos ao fato de que, uma vez que não se conhece outra forma de resolver o problema, essa foi adotada. No descritor 10, os registros de representação privilegiados foram o simbólico, o tabular, o figural e em língua natural.

Para concluir, foi possível observar que em se tratando dos registros de representação, as transformações desses, não são empregadas totalmente de acordo com as ideias presentes na teoria de Duval, em que os processos cognitivos são compreendidos por meio de tais mecanismos, ou seja, em que se cabe afirmar que o sujeito conhece um objeto quando transita entre suas diferentes representações, normalmente, por meio de conversões.

Pudemos perceber que as representações e suas transformações (tratamentos e conversões) adquirem uma característica de ferramenta de apoio para a resolução de problemas combinatórios. Isso ocorre porque os mecanismos de justificação e comprovação da solução exigem a mobilização das diferentes representações, porém, com a finalidade de obter uma resposta em língua natural para um problema que é (normalmente) enunciado nesse mesmo tipo de registro.

Portanto, as diferentes representações semióticas (tabular, simbólica, gráfica, entre outras) mobilizadas para explorar um ente matemático podem adquirir o propósito de apreensão das características e comportamentos desse objeto. De outro modo, esses registros podem ser mobilizados devido à necessidade de justificação um procedimento adotado para resolver um problema que necessita, por exemplo, do emprego do raciocínio combinatório.

\section{Considerações}

Ao concluir este estudo, consideramos que as orientações de Batanero et tal (1996, 1997, 2001 e 2003) foram imprescindíveis para compreender o raciocínio combinatório e, consequentemente, para estabelecer os descritores que apontaram indícios para analisar as produções dos estudantes a fim de identificar se, e como, os registros de representação semiótica foram mobilizados ao resolver atividades de contagem.

Das estratégias identificadas, concluímos que a regra do produto (princípio multiplicativo) foi a mais empregada, seguida da formação de configurações solicitadas no enunciado e decomposição do problema inicial. Esses e outros modos de resolver atividades que requerem o raciocínio combinatório foram utilizados de forma isolada ou em associação, ou seja, algumas resoluções apresentam mais de uma estratégia simultaneamente e estão presentes em 22 (vinte e duas) das 40 (quarenta) atividades analisadas.

A análise por meio dos descritores permitiu identificar 30 (trinta) atividades do tipo seleção (escolha de uma amostra a partir de um conjunto de objetos), seis do tipo partição (ordenação de objetos em caixas, células, urnas, etc.) e quatro do tipo 
distribuição (divisão de objetos de um conjunto em subconjuntos). Na resolução das questões, foi possível observar a mobilização dos registros tabular, em árvore, figural e, principalmente, simbólico. Enquanto isso, a reposta à pergunta do problema foi apresentada predominantemente por meio dos registros em língua natural ou simbólico.

Vale ainda ressaltar que esta pesquisa se mostra importante por ampliar os mapeamentos de estudos que apresentam referencial teórico nos registros de representação semiótica, por exemplo, os encontrados em Colombo, Flores e Moretti (2008); Curi, Ferreira e Santos (2013) e Boemo, Mariani e Rosa (2014). Também é necessária por apresentarmos um mapeamento de pesquisas sobre o raciocínio combinatório e por auxiliar na compreensão de como os alunos de ensino médio mobilizam registros de representação ao resolver atividades que necessitam esse tipo de raciocínio.

Por fim, ressaltamos que os resultados desta pesquisa poderiam ser complementados por outros estudos que investigassem a compreensão que o estudante possui sobre as estratégias de resolução de problemas combinatórios.

\section{Referências}

ALBUQUERQUE, R. S. A. C. Uma investigação no ensino médio sobre o raciocínio combinatório e a divergência de resultados na resolução de problemas de contagem. 2014 . p. 172. Dissertação (Mestrado Profissional em Ensino de Ciências Exatas). Centro Universitário Univates, Lajeado, 2014.

BATANERO, M. C.; GODINO, J. D.; NAVARRO-PELAYO, V. Razionamento Combinatorio. Madrid: Síntesis, 1996.

BATANERO, M. C.; GODINO, J. D.; NAVARRO-PELAYO, V. Combinatorial Reasoning and its assessment. In: Gal, I. \& Garfield, J. B. (editors), The Assessment Challenge in Statistics Education. Amsterdam: International Statistical Institute \& I.O.S. Press, 1997, p. 239-252. Disponível em:

$<$ https://www.stat.auckland.ac.nz/ iase/publications/assessbk/chapter18.pdf>. Acesso em: 16 mar. 2016.

BICUDO, Maria A. V. Meta-análise: seu significado para a pesquisa qualitativa. REVEMAT: Revista Eletrônica de Educação Matemática, Florianópolis - SC, v. 9, Ed. Temática (junho), p. 07-20, 2014.2 Disponível em: <https://periodicos.ufsc.br/index.php/revemat/article/viewFile/1981-1322.2014v9nespp7/27377>. Acesso em: 4 jan. 2016.

BOEMO, M. S. ,ROSA, C. W., E MARIANI, R. C. P. Os Registros de Representação Semiótica nas Pesquisas em Matemática: um olhar para os sistemas lineares e funções. In: ESCOLA DE INVERNO DE EDUCAÇÃO MATEMÁTICA, 4, 2014, Santa Maria. Anais eletrônicos... Santa Maria, UFSM, 2014 Os Registros de Representação Semiótica nas Pesquisas em Matemática: Um olhar para os sistemas lineares e funções. Disponível em: <http://w3.ufsm.br/ceem/eiemat/Anais/arquivos/ed_4/CC/CC_Boemo_Marinela.pdf>. Acesso em: 10 ago. 2016.

BRASIL, Ministério da Educação. Secretaria da Educação Média e Tecnológica. PCN+ Ensino Médio: Orientações Educacionais Complementares aos Parâmetros Curriculares Nacionais. Brasília: MEC/Semtec, 2002. 
COLOMBO, J. A. A.; FLORES, C. R.; MORETTI, M. T. Registros de Representação Semiótica nas Pesquisas Brasileiras em Educação Matemática: pontuando tendências. ZETETIKÉ - Cempem, FE. Unicamp, v. 16, n. 29, jan./jun. 2008.

CURI, E.; FERREIRA, F. A.; SANTOS, C. A. B. Um cenário sobre pesquisas brasileiras que apresentam como abordagem teórica os registros de representação semiótica. EM TEIA - Revista de Educação Matemática e Tecnológica Iberoamericana. v. 4, n. 2, 2013.

DUVAL, R. Registros de representações semióticas e funcionamento cognitivo da compreensão em Matemática. In: MACHADO, Silvia Dias Alcântara Aprendizagem Matemática: Registros de Representação Semiótica. Campinas: Papirus, 2003.

DUVAL, R. Semiósis e pensamento humano: Registros semióticos e aprendizagens intelectuais. Trad. Lênio Fernandes Levy e Marisa Rosâni Abreu da Silveira. São Paulo: Editora Livraria da Física, 2009.

DUVAL, R. Abordagem cognitiva de problemas de Geometria em termos de congruência. Tradução: Méricles Thadeu Moretti. REVEMAT. Florianópolis, v.7, n.1, p.118-138, 2012a.

DUVAL, R. Registros de representação semiótica e funcionamento cognitivo do pensamento. Tradução: MériclesThadeu Moretti. REVEMAT. Florianópolis, v.7, n.2, p.266-297, 2012b.

FONSECA, G. A. M. Análise Combinatória na Educação de Jovens e Adultos: uma proposta de ensino a partir da resolução de problemas. 2012. 178 p. Dissertação (Mestrado em Ensino de Matemática). Universidade Federal do Rio Grande do Sul, Porto Alegre, 2012.

GUZMÁN, R. R.; BATANERO, M. C.; GODINO, J. D. Dificultad de los problemas combinatorios en estudiantes con preparación matemática avanzada. Números revista de didáctica de las matemáticas, Espanha, v. 47, set. 2001, p. 33-47. Disponível em:

<http://www.sinewton.org/numeros/numeros/47/Articulo03.pdf>. Acesso em: 16 mar. 2016.

GUZMÁN, R. R.; BATANERO, M. C.; GODINO, J. D. Estrategias generales y estrategias aritméticas em la resolución de problemas combinatorios. Educación Matemática, v. 15, n. 2, 2003, p. 05-26. Disponível em: < http://www.redalyc.org/pdf/405/40515201.pdf>. Acesso em: 16 mar. 2016.

FIORENTINI, D.; LORENZATO, S. Investigação em Educação Matemática: percursos teóricos e metodológicos. 1aㅡ ed. Campinas: Autores Associados, 2006.

NAVARRO-PELAYO, V.; BATANERO, M. C.; GODINO, J. D.; Razonamiento combinatorio en alumnos de secundaria. Educación Matemática, Espanha v. 8 (1), 1996, p. 26-39. Disponível em: $<$ http://www.ugr.es/ batanero/pages/ARTICULOS/RAZON.pdf>.

Acesso em: 16 mar. 2016.

PESSOA, C.; BORBA, R. O raciocínio combinatório do início do ensino fundamental ao término do ensino médio. In: X ENCONTRO NACIONAL DE EDUCAÇÂO MATEMÀTICA, 2010, Salvador/BA. Anais... Salvador/BA: Universidade Católica do Salvador, 2010. Disponível em: <http:// http://www.gente.eti.br/lematec/CDS/ENEM10/>. Acesso em: 26 fev. 2016.

PINHEIRO, C. A. M. O Ensino de Análise Combinatória a partir de Situações-Problema. 2008. 166 p. Dissertação (Mestrado em Educação). Universidade Estadual do Pará, Belém, 2008.

SOUZA, A. C. P. Análise combinatória no ensino médio apoiada na metodologia de ensinoaprendizagem-avaliação de Matemática através da resolução de problemas. 2010. $344 \mathrm{p}$. Dissertação (Mestrado em Educação Matemática). Universidade Estadual Paulista - Campus de Rio Claro, Rio Claro, 2010.

SCHMIDT, W. Raciocínio combinatório: uma meta-análise a partir dos registros de representação semiótica. 2016. 130 p. Dissertação (Mestrado em Educação Matemática). Universidade Federal de Santa Maria - Santa Maria, 2016.

Submissão: 02/11/2016

Aceite: 05/09/2017 